\title{
Clinical pathways for urology patients during the COVID-19 pandemic
}

\author{
Alchiede SIMONATO 1, 2, Gianluca GIANNARINI ${ }^{3}$, Alberto ABRATE 1, \\ Riccardo BARTOLETTI ${ }^{4}$, Alessandro CRESTANI ${ }^{5}$, Cosimo DE NUNZIO 6 , \\ Andrea GREGORI 7, Giovanni LIGUORI ${ }^{8}$, Giacomo NOVARA 9 , \\ Nicola PAVAN ${ }^{8}$, Carlo TROMBETTA ${ }^{8}$, Andrea TUBARO ${ }^{6}$, Francesco PORPIGLIA ${ }^{10}$, \\ Vincenzo FICARRA $11 *$ on behalf of the Research Urology Network (RUN)
}

${ }^{1}$ Section of Urology, Department of Surgical, Oncological and Oral Sciences, University of Palermo, Palermo, Italy; ${ }^{2}$ Unit of Urology, Department of Surgery, S. Croce e Carle Hospital, Cuneo, Italy; ${ }^{3}$ Unit of Urology, Santa Maria della Misericordia Academic Medical Center, Udine, Italy; ${ }^{4}$ Unit of Urology, Department of Translational Research and New Technologies, University of Pisa, Pisa, Italy; ${ }^{5}$ Unit of Urology, Veneto Institute of Oncology IOV - IRCCS, Padua, Italy; 'Department of Urology, Sant'Andrea Hospital, Sapienza University, Rome, Italy; ${ }^{7}$ Unit of Urology, ASST Fatebenefratelli-Sacco, Sacco Hospital, Milan, Italy; ${ }^{8}$ Department of Urology, Cattinara Hospital, University of Trieste, Trieste, Italy; ${ }^{9}$ Department of Surgery, Oncology, and Gastroenterology, Urology Clinic, University of Padua, Padua, Italy; ${ }^{10}$ Division of Urology, Department of Oncology, School of Medicine, San Luigi Hospital, University of Turin, Orbassano, Turin, Italy; ${ }^{11}$ Section of Urology, Gaetano Barresi Department of Human and Pediatric Pathology, University of Messina, Messina, Italy

*Corresponding author: Vincenzo Ficarra, Section of Urology, Gaetano Barresi Department of Human and Pediatric Pathology, G. Martino Polyclinic Hospital, Via Consolare Valeria 1, 98125 Messina, Italy. E-mail: vficarra@unime.it

\section{A B S T R A C T}

The public health emergency caused by the Coronavirus Disease 2019 (COVID-19) pandemic has resulted in a significant reallocation of health resources with a consequent reorganization of the clinical activities also in several urological centers. A panel of Italian urologists has agreed on a set of recommendations on pathways of pre-, intra- and post-operative care for urological patients undergoing urgent procedures or non-deferrable oncological interventions during the $\mathrm{CO}$ VID-19 pandemic. Simplification of the diagnostic and staging pathway has to be prioritized in order to reduce hospital visits and consequently the risk of contagion. In absence of strict uniform regulations that impose the implementation of nasopharyngeal swabs, we recommend that an accurate triage for COVID-19 symptoms be performed both by telephone at home before hospitalization and at the time of hospitalization. We recommend that during hospital stay patients should be provided with as many instructions as possible to facilitate their return to, and stay at, home. Patients should be discharged under stable good conditions in order to minimize the risk of readmission. It is advisable to reduce or reschedule post-discharge controls and implement an adequate system of communication for telemonitoring discharged patients.

(Cite this article as: Simonato A, Giannarini G, Abrate A, Bartoletti R, Crestani A, De Nunzio C, et al.; the Research Urology Network (RUN). Clinical pathways for urology patients during the COVID-19 pandemic. Minerva Urol Nefrol 2020;72:37683. DOI: $10.23736 / \mathrm{S} 0393-2249.20 .03861-8$ )

KEY wORDS: Coronavirus; COVID-19; Clinical pandemic; Urology; Practice guideline; Clinical pathways; Telemedicine.

$\mathrm{T}$ he public health emergency caused by the Coronavirus Disease 2019 (COVID-19) pandemic has resulted in a significant reallocation of health resources with a consequent reorganization of the clinical activities also in several urological centers based on the availability of hospital beds, and above all, anesthesiologists. Recently, the Research Urology Network (RUN) proposed a document to guide the reorganization of urological procedures during the COVID-19 pandemic, 


\section{COPYRIGHT $^{\circledR} 2020$ EDIZIONI MINERVA MEDICA}

identifying non-deferrable, semi-non-deferrable and deferrable interventions. ${ }^{1}$ Other recommendations on the same topic have been proposed by different experts and international scientific societies. ${ }^{2-4}$ However, all the documents currently available do not provide sufficient operational details on the initial management of urological patients as well as on their post-treatment care during the COVID-19 pandemic. The need to reduce patient accesses to hospitals and to use the available diagnostic resources in the most rational way generates the proposal for simplified diagnostic pathways based both on good clinical practice and on the clinical experience of all authors involved in drafting of the present document. Obviously, these pathways must guarantee quality standards that allow early diagnosis and appropriate treatment of all possible complications related to the different urological procedures/surgeries performed. Furthermore, discharge from hospitals should be a safe event not only for patients, but also for the entire community.

The aim of this document is to provide the international urological community with a valid aid for the reorganization of urological activities during, and immediately after, the COVID-19 pandemic. This article is focused on the analysis of the measures to be adopted in all different phases of the pathway of care for urological patients in the times of the COVID-19 pandemic, from pre-admission and hospitalization to intraand postoperative phase and follow-up with essential recommendations provided at discharge. These recommendations were discussed and shared within a team of national experts affiliated to the Research Urology Network (RUN).

This document was drafted based on the few data available in urology literature on the management of urological patients in the COVID-19 pandemic era. A simplification of the diagnostictherapeutic algorithms for the main urgent urological conditions was agreed upon considering the recommendations of the 2020 European Association of Urology (EAU) guidelines ${ }^{5}$ and based on the experience gained by the authors in the emergency phase of the COVID-19 pandemic in Italy. After electronically sharing the main objectives of the document, a first draft was cre- ated which was subsequently shared, commented on and modified on the basis of a conference call made between the authors on March 28th 2020. The final version of the manuscript was completed on March 29th 2020 after approval by all authors.

The full Italian version of this document can be found in Supplementary Digital Material 1 (Supplementary File 1).

\section{Pathways for emergency urological conditions}

Based on recently published documents, 1,2 the main urological emergency conditions to be discussed are:

- upper urinary tract obstruction (with or without sepsis);

- acute or chronic urinary retention;

- gross hematuria;

- genitourinary trauma and acute scrotum.

The decisional algorithm shown in Figure 1 summarizes the pathway to follow in case of obstruction of the upper urinary tract with or without sepsis.

In particular, if the emergency department does not allow for, or deems risky, the execution of an abdominal-pelvic CT due to organizational or logistical reasons, it is proposed to simplify the diagnostic process with exclusively an abdominal-pelvic ultrasound and to plan the abdominal-pelvic CT (with or without contrast medium) after the COVID-19 pandemic.

The decisional algorithm shown in Figure

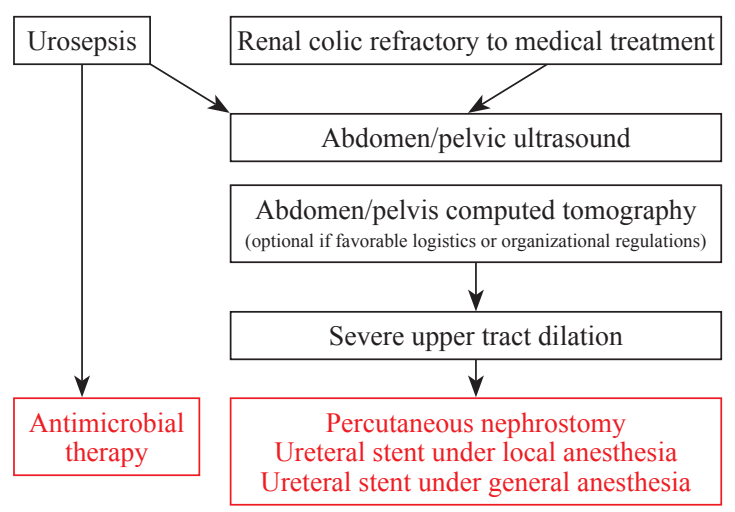

Figure 1.-Decisional algorithm for diagnosis and management of upper urinary tract obstruction (with or without sepsis) during the COVID-19 pandemic. 


\section{COPYRIGHT $^{\odot} 2020$ EDIZIONI MINERVA MEDICA}

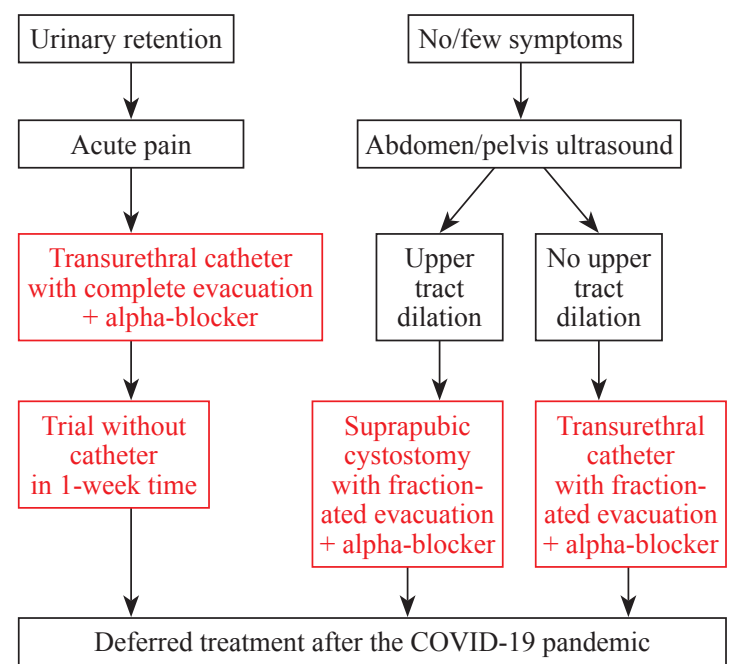

Figure 2.-Decisional algorithm for the management of urinary retention during the COVID-19 pandemic.

2 summarizes the pathway to follow in case of acute or chronic urinary retention. Patients with acute urine retention can be immediately treated with transurethral catheter without the need for fractional emptying. In patients with chronic urine retention, the choice between suprapubic cystostomy and transurethral catheter is related to the presence of an upper urinary tract dilatation or the impossibility to catheterize the patients due to urethral stenosis. These patients require a fractional emptying of urine to prevent the risk of ex vacuo hematuria and/or post-obstructive polyuria.

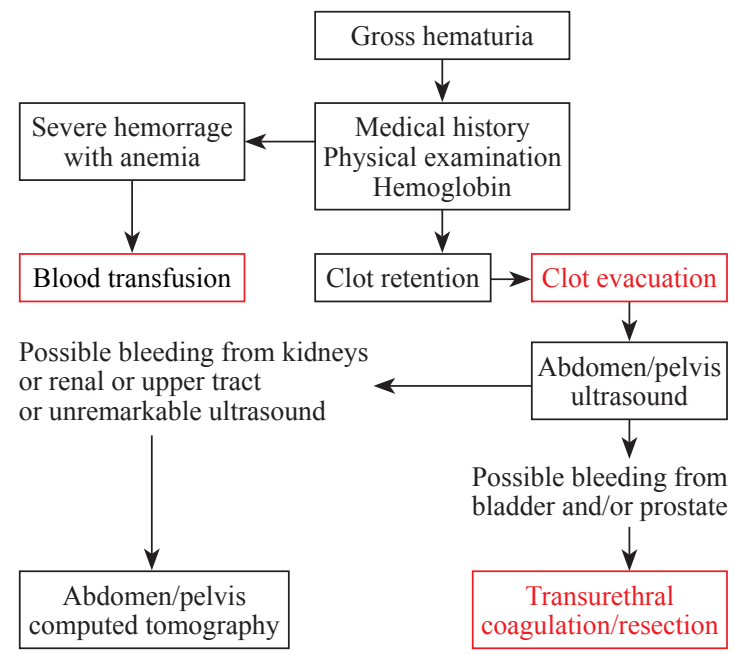

Figure 3.-Decisional algorithm for the management of gross hematuria during the COVID-19 pandemic.
Further diagnosis and treatment of the underlying pathology (such as benign prostatic obstruction) may be delayed after the emergency phase.

The decision algorithm shown in Figure 3 summarizes the pathway to follow in case of gross hematuria. It is important in these patients to minimize the risk of anemia considering the contextual shortage of blood donors observed during the COVID-19 pandemic. In case of bleeding caused by renal disease, general monitoring of the patient and the possible use of percutaneous embolization procedures, or endo-urological maneuvers such as double-J stent application, are recommended. The former does not require general anesthesia, the latter require short-duration anesthesia, but both allow to delay any surgical treatment of the underlying pathological condition to a subsequent period, whenever possible. These maneuvers prevent the development of severe anemia and the onset of possible obstructions from clots with consequent risk of urosepsis.

With regard to management of genitourinary trauma and acute scrotum, the procedures during the COVID-19 pandemic must reflect the standard clinical practice.

\section{Pathways for elective surgery}

\section{Pre-admission and hospitalization}

Pre-admission and hospitalization of urological patients scheduled for elective surgery during the COVID-19 pandemic should consider two important practical aspects, such as the need to reduce patient home-hospital flow, and a limited (o null) access to all the diagnostic exams related to pre-admission.

Furthermore, it should be important to guarantee that patients coming from home do not constitute a source of contagion for hospitalized patients. For this reason, two different pathways can be outlined, one ideal and one alternative, if the former is not feasible (Figure 4).

For both pathways, preoperative examinations should be performed with a single hospital access wherever possible, after telephone triage for COVID-19 symptoms and using preferential and well-defined hospital routes. Routine blood and 


\section{COPYRIGHT $^{\circledR} 2020$ EDIZIONI MINERVA MEDICA}

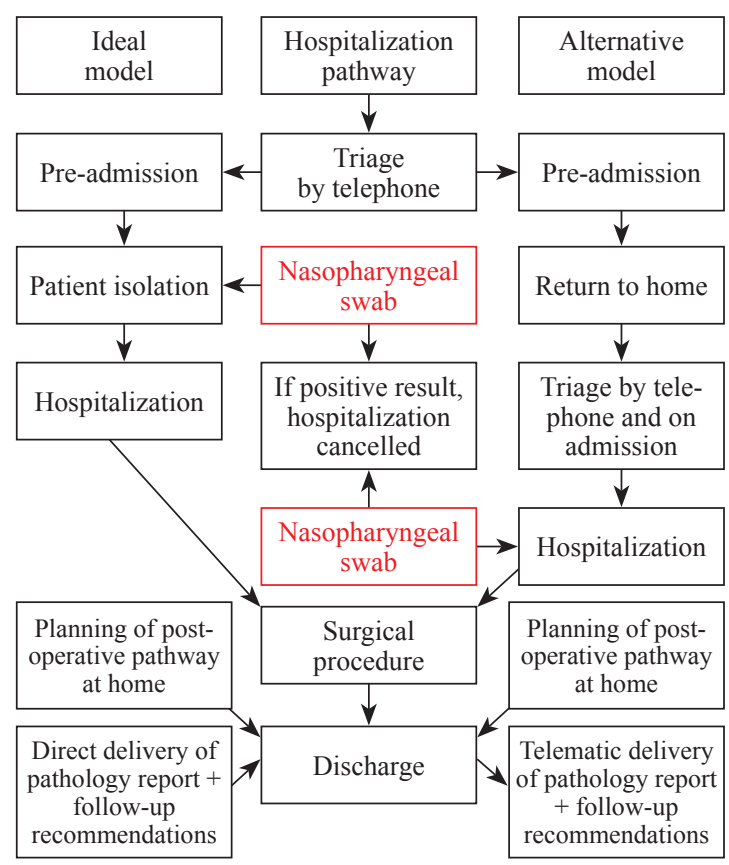

Figure 4.-Decisional algorithm for hospitalization and discharge pathways during the COVID-19 pandemic.

instrumental tests to define the anesthesiology risk should be always performed in all patients scheduled for elective surgery.

Analyzing the ideal pathway specifically, which is desirable in COVID-19-free hospitals, during the pre-admission phase it would be advisable to carry out a nasopharyngeal swab in all patients to rule the presence of the severe acute respiratory syndrome coronavirus 2 (SARSCoV-2) out. Test results should be available within a day. At the end of the pre-admission workup, each patient waiting for test results should be isolated in a single room within a dedicated area. After receiving a negative test result, the patient should be sent directly to the hospital ward. In the event of a positive test result in an asymptomatic subject, the patient must be isolated at home and hospitalization has to be postponed.

The alternative pathway, which should be implemented if pre-admission and hospitalization cannot be completed in a single step, dictates, instead, that triage, first carried out by telephone and subsequently at the time of patient access to the ward, is repeated strictly at each hospital access, and that a nasopharyngeal swab for SARS$\mathrm{CoV}-2$ is performed before surgery.
In accordance with the indications of the Centers for Disease Control and Prevention, ${ }^{6}$ it is advisable to guarantee a single access point to facilitate screening procedures.

Excluding the risk of any suspicious symptoms and signs (body temperature) of COVID-19, hospitalized patients should be asked to wear a surgical mask and to observe the hygiene rules recommended for the general population. A valid aid is to reduce the number of beds per room and/or to ensure the minimum safety distance between patients.

The recommendation to use individual protection systems is mandatory both for patients and for healthcare workers.

Considering that most non-deferrable surgical procedures are performed for the treatment of genitourinary malignancies, ${ }^{1}$ staging is of utmost importance. In this context, although adherence to recommendations of international guidelines should be respected, those examinations that are not essential for surgical planning and staging should be postponed (Table I).

\section{Intraoperative and postoperative phase}

\section{Intraoperative phase}

As previously reported, we recommend that surgical procedures be performed by experienced operators using those approaches and techniques they are more confident with, in order to decrease operating time and minimize the risk of postoperative complications. ${ }^{1}$ In the past days, contrasting opinions have emerged with regard to safety in utilization of laparoscopy procedures (conventional and robot-assisted) as a consequence of the potential risk of dissemination of Coronavirus via laparoscopy gas. ${ }^{7}$ Whereas the Intercollegiate General Surgery Guidance recommend that laparoscopy should not be used, ${ }^{3}$ guidelines from EAU Robotic Urology Section (ERUS) provide a list of non-deferrable or semi-non-deferrable robot-assisted procedures that should be performed based on the different impact of the COVID-19 pandemic at different hospitals. ${ }^{4}$ While we await more data and recommendations based on the evidence of a concrete risk of viral contamination during laparoscopy procedures, we confirm our advice to use laparoscopy procedures with 


\section{COPYRIGHT $^{\circledR} 2020$ EDIZIONI MINERVA MEDICA}

TABLE I.-Proposed recommendations for preoperative staging exams for the non-deferrable oncological procedures during the COVID-19 pandemic adapted from the latest version of the EAU Guidelines. ${ }^{5}$ The panel suggest that in case of indeterminate findings on adapted staging or in complex cases, the EAU Guidelines be fully followed.

\begin{tabular}{|c|c|c|c|}
\hline Condition & Surgical procedure & 2020 EAU Guidelines & $\begin{array}{l}\text { Adaptation to the } \\
\text { COVID-19 pandemic }\end{array}$ \\
\hline $\begin{array}{l}\text { Muscle-invasive bladder } \\
\text { cancer }\end{array}$ & $\begin{array}{l}\text { Radical cystectomy with } \\
\text { urinary diversion }\end{array}$ & $\begin{array}{l}\text { - Chest/abdomen/pelvis CT } \\
\text { - CT-urography }\end{array}$ & - Chest/abdomen/pelvis CT \\
\hline $\begin{array}{l}\text { Refractory urothelial } \\
\text { carcinoma in situ of the } \\
\text { bladder }\end{array}$ & & $\begin{array}{l}\text { - Diagnostic ureteroscopy* } \\
\text { - Abdomen/pelvic MRI* }\end{array}$ & \\
\hline $\begin{array}{l}\text { Bladder cancer with high risk } \\
\text { of progression }\end{array}$ & $\begin{array}{l}\text { Transurethral resection of } \\
\text { bladder tumors }\end{array}$ & $\begin{array}{l}\text { - CT-urography (selected } \\
\text { cases) }\end{array}$ & $\begin{array}{l}\text { - CT-urography (selected cases } \\
\text { at first diagnosis) }\end{array}$ \\
\hline $\begin{array}{l}\text { High-risk upper urinary tract } \\
\text { urothelial carcinoma }\end{array}$ & $\begin{array}{l}\text { Nephroureterectomy with } \\
\text { possible template-based } \\
\text { lymph node dissection }\end{array}$ & $\begin{array}{l}\text { - CT-urography } \\
\text { - Diagnostic ureteroscopy* } \\
\text { - Urethrocystoscopy }\end{array}$ & $\begin{array}{l}\text { - CT-urography } \\
\text { • Flexible urethrocystoscopy }\end{array}$ \\
\hline $\begin{array}{l}\text { High-risk localized or locally } \\
\text { advanced prostate cancer }\end{array}$ & $\begin{array}{l}\text { Radical prostatectomy with } \\
\text { template-based pelvic lymph } \\
\text { node dissection }\end{array}$ & $\begin{array}{l}\text { - Abdomen/pelvis CT or MRI } \\
\text { - Bone scan } \\
\text { - Multiparametric prostate } \\
\text { MRI* }\end{array}$ & $\begin{array}{l}\text { - Abdomen/pelvis CT } \\
\text { - Bone scan }\end{array}$ \\
\hline Renal cell carcinoma cT3-4 & $\begin{array}{l}\text { Radical nephrectomy (with } \\
\text { caval thrombus removal) }\end{array}$ & $\begin{array}{l}\text { - Chest/abdomen CT } \\
\text { - Abdomen CEUS/MRI* }\end{array}$ & - Chest/abdomen CT \\
\hline Renal cell carcinoma cT2 & $\begin{array}{l}\text { Radical nephrectomy } \\
\text { Partial nephrectomy (selected } \\
\text { cases) }\end{array}$ & $\begin{array}{l}\text { - Chest/abdomen CT } \\
\text { - Abdomen CEUS/MRI* }\end{array}$ & - Chest/abdomen CT \\
\hline Testicular cancer & Radical orchidectomy & $\begin{array}{l}\text { - Serum tumor markers } \\
\text { - Chest/abdomen/pelvis CT } \\
\text { - Brain MRI/TC (selected } \\
\text { cases) }\end{array}$ & $\begin{array}{l}\text { - Serum tumor markers } \\
\text { - Chest/abdomen/pelvis CT }\end{array}$ \\
\hline Penile cancer & $\begin{array}{l}\text { Penectomy } \\
\text { Inguinal lymph node } \\
\text { dissection }\end{array}$ & $\begin{array}{l}\text { - Pelvic CT or PET/CT* } \\
\text { - Penile doppler US/MRI* } \\
\text { - Chest/abdomen/pelvis CT } \\
\text { in N+ }\end{array}$ & $\begin{array}{l}\text { - Pelvic CT } \\
\text { - Chest/abdomen/pelvis CT } \\
\text { in } \mathrm{N}+\end{array}$ \\
\hline
\end{tabular}

CT: computed tomography; MRI: magnetic resonance imaging; CEUS: contrast-enhance ultrasound; EAU: European Association of Urology; COVID-19: Coronavirus Disease 2019; PET: positron-emission tomography.

*Optional/weak strength rating.

TABLE II.-Proposed intraoperative measures to decrease the risk of viral contamination during laparoscopy procedures (modified from the Society of American Gastrointestinal and Endoscopic Surgeons). ${ }^{7}$

Intraoperative measures

1. Repeatedly clean surgical instruments from blood or other body fluids

2. Avoid formation of collections of blood or other body fluids at the level of trocars caused by gas leak

3. Use suction to remove surgical smokes and renovate gas

4. Avoid two-way pneumoperitoneum insufflators

5. Keep pneumoperitoneum and insufflation pressure as low as possible

6. Keep Trendelenburg grade at a minimum

7. Minimize pneumoperitoneum-related effects on cardio-pulmonary function

8. Set electrocautery power at the lowest level

9. Avoid prolonged surgical dissection to decrease smoke formation

10. Continuously check for breakage of protective equipment for both instruments and operating staff

extreme caution, and to adopt all measures for minimizing the risk of viral contagion to operating staff (Table II). ${ }^{7}$ Figure 5 shows the use of a device for suction of surgical smokes produced by electrocautery during open surgery, which can be also used during laparoscopy.

\section{Postoperative phase}

In order to decrease complications and hospital stay, Enhanced Recovery After Surgery protocols should be adopted. ${ }^{8}$

Among the measures adopted to contain the 


\section{COPYRIGHT $^{\odot} 2020$ EDIZIONI MINERVA MEDICA}
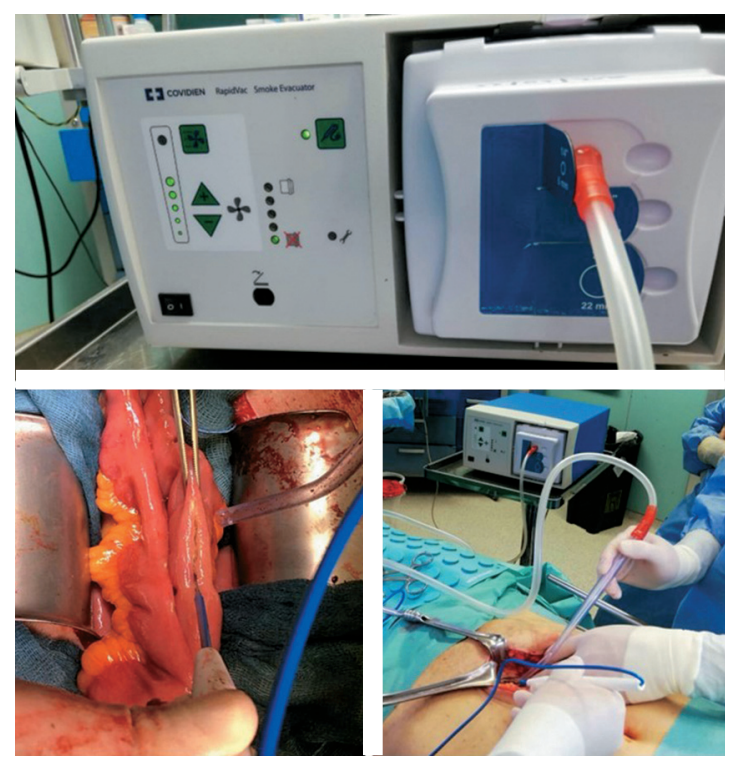

Figure 5.- Rapidvac $^{\mathrm{TM}}$ Smoke Evacuator is a medical device that allows for the immediate suction of surgical smokes produced by electrocautery during open (and laparoscopic) surgery (courtesy of Prof. Francesco Porpiglia).

risk of viral contagion, many hospitals have prohibited access to the ward to patient relatives. This requires the implementation of a system that allows for daily transmission of information on clinical course to patient relatives.

In case of suspected COVID-19 patients in the ward, hospital authorities should be immediately informed in order to adopt appropriate isolation measures, perform diagnostic tests and, eventually, transfer the patients to dedicated COVID-19 areas in the hospital. It is equally fundamental that medical or nurse staff that have come in contact with COVID-19 patients undergo nasopharyngeal swabs or, alternatively, follow the regulations imposed by local hospital authorities that may vary depending on different logistics and organizational protocols. In any case, standard postoperative therapeutic pathways should not be modified.

Patients following urological surgical procedures should be discharged only when their clinical conditions and logistics allow for a safe return to their home, thus reducing the risk of occurrence of immediate complications and readmission.

Scheduling of postoperative pathway and follow-up visits represents a critical issue to be considered especially in the emergency phase of the COVID-19 pandemic. Patients with indwelling urinary catheters (nephrostomy tube, suprapubic cystostomy tube or transurethral catheter) should be informed and trained for a completely autonomous home care. With regard to this, photographic or video tutorials could be of aid to ease home care of urinary stoma for patients and their relatives. Patients discharged with indwelling internal ureteral stents need no specific advice. Moreover, using absorbable material for skin suture avoids subsequent hospital visits for their removal.

Table III, IV summarize the main variations in follow-up schedule that should be followed during the COVID-19 pandemic compared to standard conditions for urgent and oncological non-deferrable urological procedures, respectively. We believe it is useful that ward staff provide as many instructions as possible during the hospital stay in order to facilitate home care. Among these explanations, it is particularly important to inform patients and their relatives that potential vehicles of viral transmission are feces and blood besides nasopharyngeal fluids and saliva. ${ }^{1}$

The need for reduction of postoperative out-

TABLE III.-Proposed recommendations on discharge after urgent procedures during the COVID-19 pandemic compared to standard health conditions.

\begin{tabular}{|c|c|c|c|c|c|c|c|c|}
\hline \multirow{2}{*}{ Recommendation } & \multicolumn{2}{|c|}{ Renal trauma } & \multicolumn{2}{|c|}{ Testicular torsion } & \multicolumn{2}{|c|}{ Infection/abscess } & \multicolumn{2}{|c|}{ Priapism } \\
\hline & Standard & COVID-19 & Standard & COVID-19 & Standard & COVID-19 & Standard & COVID-19 \\
\hline Skin suture removal & Yes & No a & No a & No a & Yes & No a & No a & No a \\
\hline Surgical wound check & Yes & No & Yes & No & Yes & No & Yes & No \\
\hline Blood tests & Yes & No ${ }^{b}$ & No & No & Yes & No & No & No \\
\hline Telemonitoring & No & Yes & No & Yes & No & Yes & No & Yes \\
\hline
\end{tabular}

COVID-19: Coronavirus Disease 2019.

a Absorbable material is recommended; ${ }^{\mathrm{b}}$ discharge under stable good conditions. 


\section{COPYRIGHT $^{\circledR} 2020$ EDIZIONI MINERVA MEDICA}

TABLE IV.- Proposed recommendations on discharge after non-deferrable oncological procedures during the COVID-19 pandemic compared to standard health conditions.

\begin{tabular}{|c|c|c|c|c|c|c|c|c|c|c|}
\hline \multirow{2}{*}{ Recommendation } & \multicolumn{2}{|c|}{$\begin{array}{c}\text { Radical } \\
\text { cystectomy }\end{array}$} & \multicolumn{2}{|c|}{$\begin{array}{c}\text { Radical } \\
\text { prostatectomy }\end{array}$} & \multicolumn{2}{|c|}{$\begin{array}{c}\text { Radical } \\
\text { nephrectomy }\end{array}$} & \multicolumn{2}{|c|}{$\begin{array}{c}\text { Radical } \\
\text { orchidectomy }\end{array}$} & \multicolumn{2}{|c|}{ Penectomy } \\
\hline & Standard & COVID-19 & Standard & OVID-19 & Standar & PVID-19 & Standar & VID-19 & Standar & DVID-19 \\
\hline Skin suture removal & Yes & No ${ }^{a}$ & Yes & $\mathrm{No}^{a}$ & Yes & No ${ }^{a}$ & No & No ${ }^{a}$ & No & No ${ }^{a}$ \\
\hline Surgical wound check & Yes & No & Yes & No & Yes & No & Yes & No & Yes & No \\
\hline $\begin{array}{l}\text { Bladder catheter } \\
\text { removal after } \\
\text { cystography }\end{array}$ & Yes & $\begin{array}{l}\text { Yes/no } \\
\text { (neo- } \\
\text { bladder) }\end{array}$ & Yes/no & $\mathrm{No}^{\mathrm{b}}$ & No & No & No & No & No & No \\
\hline $\begin{array}{l}\text { Ureteral catheter } \\
\text { removal }\end{array}$ & Yes/no ${ }^{c}$ & No & No & No & No & No & No & No & No & No \\
\hline Serum tumor markers & No & No & No & No & No & No & Yes & Yes & No & No \\
\hline Blood tests & Yes & No d & No & No & Yes & No d & No & No & No & No \\
\hline Telemonitoring & No & Yes & No & Yes & No & Yes & No & Yes & No & Yes \\
\hline
\end{tabular}

COVID-19: Coronavirus Disease 2019.

a Absorbable material is recommended; b removal is recommended on postoperative day 8 to 14 depending on intraoperative tissue/anastomosis conditions; c depending on type of urinary diversion; d to be performed only if clinical utility is expected.

patient visits requires that our centers implement new systems of telemonitoring. Unfortunately, telemedicine applications are currently still in their early phase, and the emergency generated by the COVID-19 pandemic imposes that commoner tools, such as smartphones and electronic mail, be used for telemonitoring. Urology wards should implement systems for priority communications via telephone or electronic mail from patients to the medical or nurse staff in order to check the clinical course and decrease the risk of inappropriate hospital visits.

Informing patients on the results of a pathological exam, especially after urological procedures for genitourinary malignancies that we have previously defined as non-deferrable, is one of the most critical steps in the diagnostic and therapeutic clinical path. ${ }^{1}$ Considering the lower burden of routine work for pathology personnel in these times, it would be desirable that pathology report be available before patient discharge, so that the therapeutic pathway can be completed and the follow-up program can be scheduled. If this is not an option, then we recommend that the pathological report be sent home via electronic mail being accompanied by a telephone call from the practicing urologist. This combined communication of the pathology report (electronic and telephonic) should contain all possible explanations on the oncological diagnosis and on the corresponding program with all subsequent follow-up exams or visits. Before sending all this documents, it is advisable to retrieve patient informed consent, or alternatively, follow local hospital rules.

\section{Conclusions}

The present document provides a useful set of recommendations on pathways of pre-, intraand postoperative care for urological patients undergoing urgent procedures or non-deferrable oncological interventions during the COVID-19 pandemic. Simplification of the diagnostic and staging pathway has to be prioritized in order to reduce hospital visits and consequently the risk of contagion. In absence of strict uniform regulations that impose the implementation of nasopharyngeal swabs, we recommend that an accurate triage for COVID-19 symptoms be performed both by telephone at home before hospitalization and at the time of hospitalization. We recommend that during hospital stay patients should be provided with as many instructions as possible to facilitate their return to, and stay at, home. Patients should be discharged under stable good conditions in order to minimize the risk of readmission. It is advisable to reduce or reschedule post-discharge controls and implement an adequate system of communication for telemonitoring discharged patients in order to reduce hospital visits. We finally believe that some of the solutions proposed to face the current emergency, if maintained, may optimize routine clinical practice even in standard health conditions. 


\section{COPYRIGHT $^{(\odot)} 2020$ EDIZIONI MINERVA MEDICA}

\section{References}

1. Ficarra V, Novara G, Abrate A, Bartoletti R, Crestani A, De Nunzio C, et al.; the Research Urology Network (RUN). Urology practice during the COVID-19 pandemic. Minerva Urol Nefrol 2020;72:369-75.

2. Stensland KD, Morgan TM, Moinzadeh A, Lee CT, Briganti A, Catto J, et al. Considerations in the triage of urologic surgeries during the COVID-19 pandemic. Eur Urol 2020. [Epub ahead of print]

3. The Royal College of Surgeons of Edinburgh. Intercollegiate General Surgery Guidance on COVID-19 UPDATE; 2020 [Internet]. Available from: https://www.rcsed.ac.uk/ news-public-affairs $/$ news $/ 2020 / \mathrm{march} /$ intercollegiate-general-surgery-guidance-on-covid-19-update [cited 2020, Apr 10]. 4. European Association of Urology. EAU Robotic Urology Section (ERUS) guidelines during COVID-19 emergency;
2020 [Internet]. Available from: https://uroweb.org/eau-robotic-urology-section-erus-guidelines-during-covid-19-emergency/ [cited 2020, Apr 10].

5. European Association of Urology. European Association of Urology Guidelines; 2020 [Internet]. Available from: https://uroweb.org/guidelines [cited 2020, Apr 10].

6. U.S. Center for Disease Control and Prevention. Coronavirus (COVID-19); 2020 [Internet]. Available from: https:// www.cdc.gov/coronavirus [cited 2020, Apr 10].

7. Society of American Gastrointestinal and Endoscopic Surgeons. SAGES and EAES recommendations regarding surgical response to COVID-19 crisis; 2020 [Internet]. Available from: https://www.sages.org/recommendations-surgicalresponse-covid-19 [cited 2020, Apr 10].

8. Enhanced Recovery After Surgery Society. ERAS Society Guidelines; 2020 [Internet]. Available from: https://erassociety.org/guidelines/list-of-guidelines/ [cited 2020, Apr 10].

Conflicts of interest.-The authors certify that there is no conflict of interest with any financial organization regarding the material discussed in the manuscript.

History.-Article first published online: March 30, 2020. - Manuscript accepted: March 30, 2020. - Manuscript received: March 30, 2020.

Supplementary data.-For supplementary materials, please see the HTML version of this article at www.minervamedica.it 